\title{
djb trauert um Ehrenvorsitzende Dr. Hertha Engelbrecht
}

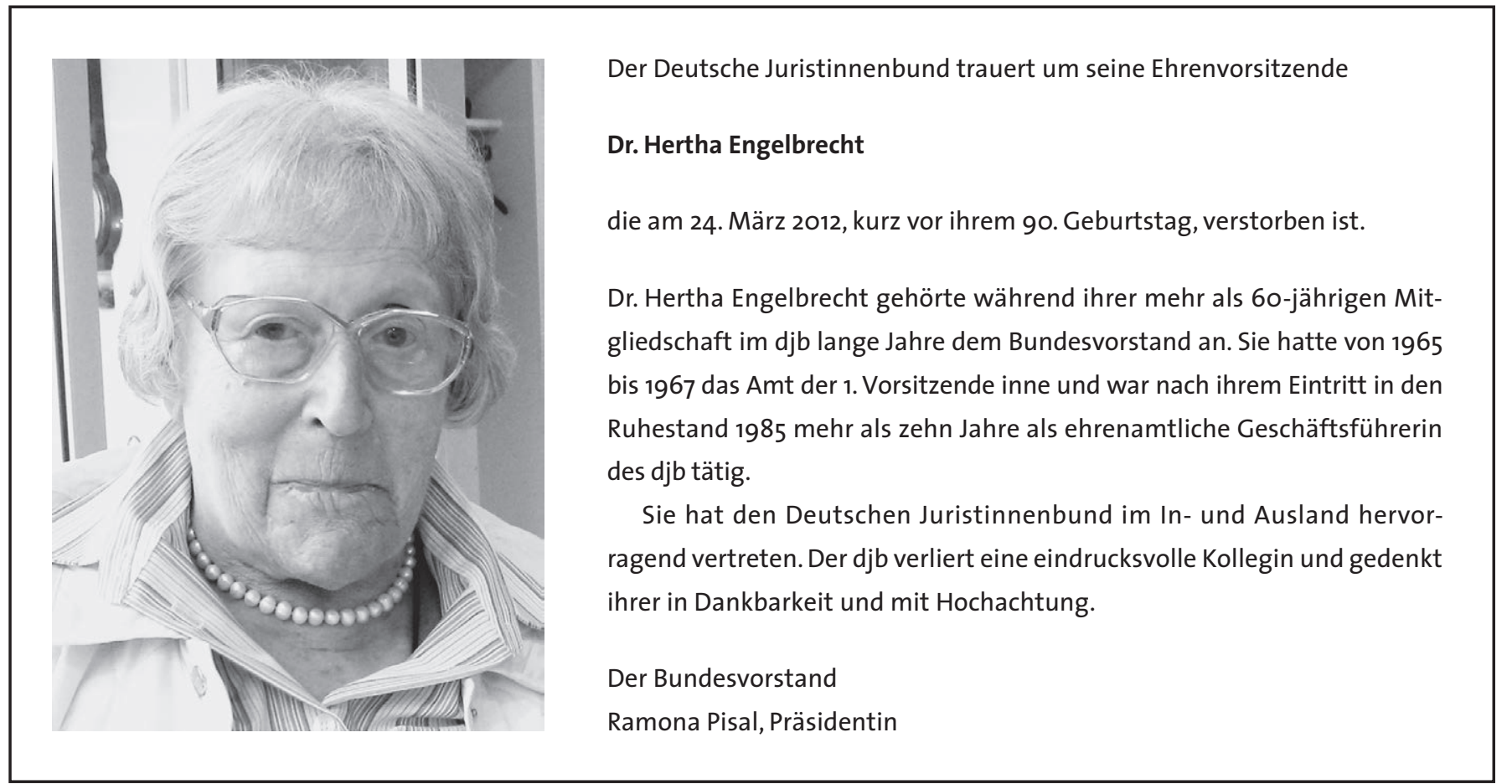

Dr. Hertha Engelbrecht wurde am 28. März 1922 in Hamburg geboren. Ihr Vater, Christian Engelbrecht, war Kaufmann. Von 1928 bis 1932 besuchte sie die Grundschule, von 1932 bis 1938 das Lyzeum Tesdorpstraße und von 1938 bis 1940 die Klosterschule in Hamburg, wo sie am 2. März 1940 ihre Reifeprüfung bestand. Nach einem Studium von Herbst 1940 bis 1942 am Dolmetscherinstitut der Heidelberger Universität legte sie am 30. Oktober 1942 mit der Note „gut“ die Prüfung als akademisch geprüfter Übersetzer (Englisch und Spanisch) ab. Bis 1945 studierte sie zwei Semester Volkswirtschaft, dann absolvierte sie ein Praktikum und war schließlich vom 1. November 1944 bis 8. Mai 1945 bei der Deutsche Vacuum Oil Company (heute: Mobil Oil AG), Hamburg, dienstverpflichtet. Von Mai 1945 bis Mai 1947 war sie als Dolmetscherin und Übersetzerin am Hamburger Oberlandesgericht tätig, das damals auch die Funktion eines Justizministeriums hatte.

Im Sommer 1947 begann sie ihr Jurastudium an der Universität Hamburg, das sie am 23. Mai 1949 mit der Ersten Juristischen Staatsprüfung „voll befriedigend“ abschloss. Es folgte ihr Referendariat am Hanseatischen Oberlandesgericht. Das Zweite Staatsexamen am 11. Dezember 1952 beendete sie ebenfalls mit „voll befriedigend“. Am 23. April 1953 wurde sie mit einer vergleichenden Arbeit im anglo-amerikanischen Recht zum Doktor der Rechte promoviert.

Vom 1. März bis 31. August 1953 war sie Assessorin in der Verwaltung der Freien und Hansestadt Hamburg, Bezirksamt Eimsbüttel. Dort schied sie wegen eines Auslandsstudiums auf eigenen Wunsch aus. Das Studienjahr 1953/54 verbrachte sie mit einem Stipendium des State Department an der Harvard Law School, Cambridge, Mass./USA. 1954 wurde sie vereidigte Dolmetscherin der englischen Sprache (Land Hamburg). Noch bis etwa 1964 war sie in Nebentätigkeit als vereidigte Dolmetscherin und Übersetzerin tätig.

Am 1. September 1954 trat sie als Assessorin i. Pr. erneut in die Verwaltung der Freien und Hansestadt Hamburg ein. Am 29. März 1955 wurde sie zur Regierungsassessorin ernannt. Am 1. Juli 1956, nach zweijähriger Assessorenzeit bei verschiedenen Bezirksämtern, der Behörde für Wirtschaft und Verkehr (ab 1. November 1955) und dem Personalamt wurde sie zur Regierungsrätin und Beamtin auf Lebenszeit befördert. Am 1. Juni 1957 wurde sie zum Rechtsamt abgeordnet. Am 15. Februar 1958 - einem Rosenmontag - wurde sie zur Senatskanzlei/Vertretung der Freien und Hansestadt Hamburg beim Bund in Bonn als Referentin abgeordnet. Sie folgte dort dem späteren Bundesverfassungsrichter Dietrich Katzenstein nach. Am 28. Mai 1958 wurde sie ganz nach Bonn versetzt und schließlich am 6. Februar 1961 zur Oberregierungsrätin ernannt. Sie wurde 1966 Vertreterin des Dienststellenleiters, am 2. Mai 1966 Regierungsdirektorin, am 1. März 1973 Leitende Regierungsdirektorin und am 8. März 1983 Senatsdirektorin. Seit dem 1. Mai 1982 bis zu ihrem Ausscheiden aus dem Dienst am 31. März 1985 auf eigenen Antrag war sie „Dienststellenleiter“. Sie war die Vertretung des Bevollmächtigten in allen die Dienststelle betreffenden Angelegenheiten. Sie koordinierte unter anderem die Facharbeit aller Referate und war Vertreterin Hamburgs im Auswärtigen, Innerdeutschen und Verteidigungsausschuss von Bundesrat und Bundestag. 
Dr. Hertha Engelbrecht war seit 1968 SPD-Mitglied. Sie war von 1964 bis 1980 Vorstandsmitglied des Deutschen Frauenrates, davon 1973 bis 1974 als Zweite Vorsitzende. Dem Frauenrat diente sie insbesondere als Justitiarin und Beraterin in Satzungs- und Namensführungsangelegenheiten. Sie nahm unter anderem an Delegationsreisen und Kongressen in Tunesien, Zaire, USA, UdSSR und Volksrepublik China teil. Von 1970 bis 1982 war sie Mitglied der Ständigen Deputation des Deutschen Juristentages und gestaltete sechs Juristentage inhaltlich mit. Von 1987 bis 1989 war sie Vorsitzende des Soroptimist Clubs Bonn-Bad Godesberg. Dort war sie auch Koordinatorin für die Stellung der Frau und Menschenrechte der Deutschen Union der Soroptimist Clubs. Am 8. Oktober 1996 wurde ihr durch den Bundespräsidenten in Berlin das Bundesverdienstkreuz 1. Klasse verliehen.

Auch im hohen Alter von 74 Jahren schwang sie sich fast täglich auf den Rücken eines Pferdes. Reisen in ferne Länder durfte sie in höherem Alter nicht mehr unternehmen und fuhr stattdessen in die Provence, wo sie sich der Aquarellmalerei widmete.

Dr. Hertha Engelbrecht trat zu einem nicht mehr belegbaren Termin bis 1955 in den Deutschen Juristinnenbund ein. Die Mitgliederversammlung des djb benannte sie je- denfalls schon am 9./10. September 1955 als Kandidatin für die Nachfolge von Dr. Schwarzhaupt in der Deutschen UNESCO-Kommission. 1962 wurde sie als Beisitzerin in den Bundesvorstand gewählt. Von 1963 bis 1965 war sie 2. Vorsitzende, von 1965 bis 1967 1. Vorsitzende des djb. Dem erweiterten Bundesvorstand gehörte sie dann von 1969 bis 1975 an. Nach ihrem Eintritt in den Ruhestand engagierte sie sich als erste Geschäftsführerin des djb; bis 1985 hatte der Verband gar keine Geschäftsstelle. Sie begann mit dem Aufbau des Bonner djb-Büros, das schnell zum Vereinsmittelpunkt wurde und als Schaltstelle zwischen den jeweils zuständigen Vorstandsmitgliedern, Kommissionsvorsitzenden und Untergruppen diente. Sie nahm Termine im Bonner Raum wahr und beschafft öffentliche Mittel für den djb. Ab 1994 wurde sie von Martina Bosch unterstützt. Dr. Hertha Engelbrecht zog sich 1997, mit 75 Jahren, aus der Verbandsarbeit zurück, nahm aber weiterhin bundesweit und auch im Raum Bonn gerne an Veranstaltungen teil und unterstützte den djb nach Möglichkeit.

Im Dezember 2011 erlitt sie einen Schlaganfall und starb am 24. März 2012 in Bonn. Sie wurde am 30. März 2012 in Gräfelfing bei München beigesetzt. (AG)

\section{Trauerrede, 30. März 2012, Gräfelfing bei München:}

Lieber Herr Engelbrecht, liebe Familien Engelbrecht und Ostertag,

zunächst möchte ich mich ganz herzlich bei Ihnen bedanken, dass sie meinen Kolleginnen im djb aus Bonn und München und mir erlauben, heute im vertrauten Kreise Ihrer Familie an der Trauerfeier für Dr. Hertha Engelbrecht und der Beisetzung ihrer sterblichen Überreste teilzunehmen. Dafür sind wir Ihnen sehr verbunden. Es war uns ein besonderes Anliegen, Dr. Hertha Engelbrecht die letzte Ehre zu erweisen und sie heute auf ihrem letzten Gang zu begleiten.

Frau Dr. Engelbrecht war uns für mehr als 60 Jahre ein treues Mitglied. Seit den Anfängen des 1948 neu gegründeten djb gehörte sie dazu und unterstützte durch ihre Mitgliedschaft und ihr aktives Eintreten für die Gleichstellung von Frauen in besonderem Maße unser gemeinsames Anliegen. Als berufstätige Frau in hervorgehobenen Positionen war sie selbst das beste Beispiel dafür, was gut ausgebildete und unabhängige Frauen ganz selbstverständlich leisten und darstellen können, wenn man sie nur lässt. Engagiert und mit sehr viel Charme und Eleganz hat sie über Jahrzehnte die Gleichberechtigung und Gleichstellung von Frauen aktiv vorangebracht.

Wir sind Dr. Hertha Engelbrecht aber weit darüber hinaus verbunden und zu besonderem Dank verpflichtet, denn sie war unsere erste Geschäftsführerin. Die zunehmende Professionalisierung unseres Verbandes erforderte neue Strukturen, und es war Dr. Hertha Engelbrecht, die sich dieser Aufgabe annahm und in Bonn in einer Einraumwohnung unsere erste Geschäftsstelle aufbaute. Sie organisierte die Verwaltung des Verbandes, koordinierte die Verbindungen zu anderen Verbänden, Ministerien und Einrichtungen, sie war die Ansprechpartnerin für die Kommissionen und die Mitglieder.

Das ist nicht nur darum besonderer Erwähnung würdig, weil sie die erste in diesem Amt war. Dr. Hertha Engelbrecht hat diese Aufgabe mit ihrem Eintritt in den Ruhestand übernommen, und sie hat sie insgesamt ehrenamtlich ausgeführt. Sie hat sehr viel Zeit und auch Geld investiert, denn ihre vielfältigen Aktivitäten für den Verband hätte sich dieser damals gar nicht leisten können. So hat sie eine Vielzahl von Terminen für den djb wahrgenommen und uns jederzeit angemessen und würdig vertreten.

Und trotzdem bleibt noch offen, was Dr. Hertha Engelbrecht für uns zu einer ganz besonderen Kollegin gemacht hat. Das war sie nämlich über ihre vielfältigen Verdienste und Zuwendungen hinaus vor allem durch ihre Persönlichkeit. Weltoffen, kompetent und charmant war sie immer und gegenüber jedem Mitglied ohne Dünkel aufmerksam, zugewandt, hilfsbereit und freundlich. Ihr stets liebenswürdiges Auftreten und ihr Lächeln hat den djb in den Bonner Jahren der ersten Geschäftsstelle nach innen und außen ganz entscheidend geprägt und repräsentiert.

Wir werden ihr Angedenken in dankbarer Erinnerung bewahren und wünschen ihr den ewigen Frieden.

Ramona Pisal, Präsidentin 